COMPATIBLE MAPPINGS AND COMMON FIXED POINTS

\author{
GERALD JUNGCK \\ Department of Mathematics \\ Bradley University \\ Peoria, Illinois 61625 \\ (Received July 30, 1985)
}

ABSTRACT. A generalization of the commuting mapping concept is introduced. Properties of this "weakened commutativity" are derived and used to obtain results which generalize a theorem by Park and Bae, a theorem by Hadzic, and others.

KEY WORDS AND PHRASES. Common fixed points, commuting mappings, compatible mappings, and $(\varepsilon, \delta)$-contractions.

1980 MATHEMATICS SUBJECT CLASSIFICATION CODE. 54H25

1. INTRODUCTION.

The following generalization of the Banach Contraction Theorem was proved in [1].

THEOREM 1.1. A continuous self map of a complete metric space $(X, d)$ has a fixed point iff there exists $r_{\varepsilon}(0,1)$ and a mapping $g: X \rightarrow X$ which commutes with $f(g f=f g)$ and satisfies: $g(x) C f(x)$ and $d(g(x), g(y)) \leq r d(f(x), f(y))$ for $x, y$ in $x$. In fact, $f$ and $g$ have a unique common fixed point.

The intent of the article [1] was to depict commuting mappings as a tool for generalization. A variety of extensions, generalizations, and applications of Theorem 1.1. which incorporated the commuting map concept followed; e.g., $[2],[3],[4],[6]-[19]$. One of the more powerful of these is the following result by Park and Bae [4] which generalizes a theorem by Meir and Keeler [5].

THEOREM 1.2. If $f$ is a continuous self map of a complete metric space $X$ and $g$ is an $(\varepsilon, \delta)$-f-contraction which commutes with $f$, then $f$ and $g$ have a unique common fixed point in $X$.

Definition 1.1. Let $f$ and $g$ be self maps of a metric space $(x, d)$. $g$ is an $(\varepsilon, \delta)$-f-contraction iff for any $\varepsilon>0$ there is a $\delta>0$ such that

(i) $\varepsilon \leq d(f(x), f(y))<\varepsilon+\delta$ implies $d(g(x), g(y))<\varepsilon$, and

(ii) $g(x)=g(y)$ when $f(x)=f(y)$

The purpose of this paper is to propose a generalization of the comuting mapping concept. Sessa [6] generalized commuting maps by calling self maps $f$ and $g$ of a metric space $(x, d)$ a weakly commuting pair iff $d(f g(x), g f(x)) \leq d(f(x), g(x))$ for $x$ in $x$. Of course, commuting pairs are weakly commuting, but the converse is false (See [6]). Baskaran and Subrahmanyam [7] and Rhoades et al. [6] obtained 
nice fixed point results using this concept. However, since elementary functions as similar as $f(x)=x^{3}$ and $g(x)=2 x^{3}$ are not weakly commutative, it is desirable to introduce a less restrictive concept - a concept we shall call compatibility.

In section 2. we define and develop properties of compatible functions, and we provide examples to illustrate the extent to which the commutativity requirement has been weakened. In section 3. we demonstrate the utility of this concept in the context of metric fixed point theory by replacing the commutativity hypothes is in Theorem 1.2. by compatibility. We further extend Theorem 1.2. by defining $(\varepsilon, \delta)$-contractibility for four functions.

In this paper, $R, R^{+}$, and $N$ shall denote the real numbers, the positive real numbers, and the positive integers respectively, with their usual topologies.

\section{COMPATIBLE MAPPINGS.}

Any pair $f$ and $g$ of self maps of a set commute on the set of common fixed points: $\{x \in X: f(x)=g(x)=x\}$. The following definition will require that $f$ and $g$ commute on the potentially larger set $\{x \in X: f(x)=g(x)\}$.

Definition 2.1. Self mappings $f$ and $g$ of a metric space $(x, d)$ are compatible iff $\lim _{n} d\left(g f\left(x_{n}\right), f g\left(x_{n}\right)\right)=0$ whenever $\left\{x_{n}\right\}$ is a sequence in $x$ such that $\lim _{n} f\left(x_{n}\right)=$ $\lim _{n} g\left(x_{n}\right)=t$ for some $t \in x$.

Clearly, any weakly commuting pair are compatible. On the other hand, the functions $f(x)=x^{3}$ and $g(x)=2 x^{3}$ are compatible since $|f(x)-g(x)|=|x|^{3} \rightarrow 0$ iff $|f g(x)-g f(x)|=6|x|^{9} \rightarrow 0$, but as noted above they are not a weakly commuting pair. (In this instance $x$ can be taken as R.)

EXAMPLE 2.1. Let $f(x)=x^{3}$ and $g(x)=2-x$ with $x=R$. $\left|f\left(x_{n}\right)-g\left(x_{n}\right)\right|=$ $\left|x_{n}-1\right|\left|x_{n}^{2}+x_{n}+2\right| \rightarrow 0$ iff $x_{n} \rightarrow 1$ and $\lim _{n}\left|f g\left(x_{n}\right)-g f\left(x_{n}\right)\right|=1 i m_{n} 6\left|x_{n}-1\right|^{2}=0$ if $x_{n} \rightarrow 1$. Thus $f$ and $g$ are compatible but are not a weakly commuting pair; e.g., let $x=0$.

EXAMPLE 2.2. Let $f(x)=2-x^{2}$ and $g(x)=x^{2}$ with $x=R$. As in Example 2.1, it is easy to show that $f$ and $g$ are compatible but not weakly comutative.

EXAMPLE 2.3. Let $f(x)=\cosh (x)$ and $g(x)=\sinh (x)$ with $x=R$. Then $|f(x)-g(x)|=e^{-x} \rightarrow 0$ iff $x \rightarrow \infty$. But $f(x), g(x) \rightarrow+\infty$ as $x \rightarrow+\infty$; i.e., $f$ and $g$ do not converge to an element $t$ of $x$. Thus the condition of compatibility is satisfied vacuously, but $f$ and $g$ do not commute.

The following observation provides a criterion for identifying compatible functions.

PROPOSITION 2.1. Let $f, g:(X, d) \rightarrow(X, d)$ be continuous, and let $F=\{x \in X:$ $f(x)=g(x)=x\}$. Then $f$ and $g$ are compatible if any one of the following conditions is satisfied.

(a) If $f\left(x_{n}\right), g\left(x_{n}\right) \rightarrow t(\varepsilon x)$, then $t \in f$.

(b) $d\left(f\left(x_{n}\right), g\left(x_{n}\right)\right) \rightarrow 0$ implies $D\left(f\left(x_{n}\right), F\right) \rightarrow 0$.

(c) $F$ compact and $d\left(f\left(x_{n}\right), g\left(x_{n}\right)\right) \rightarrow 0$ implies $D\left(x_{n}, F\right) \rightarrow 0$.

$$
(D(x, F)=\inf \{d(x, y): y \in F\} .)
$$

PROOF. Suppose that $\lim _{n} f\left(x_{n}\right)=1 i_{n} g\left(x_{n}\right)=t$ for some $t \varepsilon x$.

If $(a)$.holds, $f(t)=g(t)=t$. Then the continuity of $f$ and $g$ on $F$ imply $f g\left(x_{n}\right) \rightarrow f(t)=t$ and $g f\left(x_{n}\right) \rightarrow g(t)=t$, so that $d\left(f g\left(x_{n}\right), g f\left(x_{n}\right)\right) \rightarrow 0$ as desired. 
If (b) holds, the compatibility of $f$ and $g$ follow easily from (a) and (2.1) upon noting that $F$ is closed since $f$ and $g$ are continuous. So to complete the proof, suppose that $F$ is compact and that (2.1) and (c) hold. Then $d\left(f\left(x_{n}\right)\right.$, $\left.g\left(x_{n}\right)\right) \rightarrow 0$ and $D\left(x_{n}, F\right) \rightarrow 0$. Since $F$ is compact there is a subsequence $\left\{x_{k_{n}}\right\}$ of $\left\{x_{n}\right\}$ which converges to some element $c$ of $F$. Then $f\left(x_{k_{n}}\right) \rightarrow f(c)=c$ by the continuity of $f$ and the definition of $F$. Consequently, (2.1) implies that $c=t \in F$, so $f$ and $g$ are compatible by $(a)$.

EXAMPLE 2.4. Let $f(x)=x^{4}+a x^{2}(a \geq 1)$ and $g(x)=x^{2}$ with $x=R$. Then $\left|f\left(x_{n}\right)-g\left(x_{n}\right)\right|=x_{n}{ }^{2}\left|x_{n}{ }^{2}+(a-1)\right| \rightarrow 0$ iff $x_{n} \rightarrow 0(\varepsilon F)$, so that $f$ and $g$ are compatible by Proposition 2.1(c). But $f$ and $g$ are not weakly commutative; let $a=2, x=1$.

COROLLARY 2.1. Suppose that $f$ and $g$ are continuous self maps of $R$ such that $f-g$ is strictly increasing. If $f$ and $g$ have a common fixed point, then $f$ and $g$ are compatible.

PROOF. Immediate, since $f\left(x_{n}\right), g\left(x_{n}\right) \rightarrow t$ implies that $F=\{t\}$.

EXAMPLE 2.5. If $f(x)=x^{3}+a x$ and $g(x)=m x$ with $x=R$ and $a>m$, then $f(0)=g(0)=0$ and $f-g$ is increasing, so $f$ and $g$ are compatible by corollary 2.1. EXAMPLE 2.6. If $f(x)=e^{x}-1$ and $g(x)=x^{2} \quad(x=R), f$ and $g$ are compatible since $f(0)=g(0)=0$ and $f-g$ is increasing.

It is easy to show that if $g_{j}, f_{j}: R \rightarrow R$ and the pairs $f_{j}, g_{j}$ are compatible for $i=1,2, \ldots, n$, and if $G\left(x_{1}, x_{2}, \ldots, x_{n}\right)=\left(g_{1}\left(x_{1}\right), \ldots, g_{n}\left(x_{n}\right)\right)$ and $F\left(x_{1}, \ldots, x_{n}\right)=$ $\left(f_{1}\left(x_{1}\right), \ldots, f_{n}\left(x_{n}\right)\right)$, then $F$ and $G$ are compatible on $\left(R^{n}, d\right)$ where $d$ is the Euclidean metric. Thus the above examples show that $G(x, y)=\left(e^{x}-1,7 y\right)$ and $F(x, y)=\left(x^{2}, y^{3}+8 y\right)$ are compatible on $\left(R^{2}, d\right)$.

The following result will be useful in section 3 .

PROPOSITION 2.2. Let $f, g:(X, d) \rightarrow(X, d)$ be compatible.

1. If $f(t)=g(t)$, then $f g(t)=g f(t)$.

2. Suppose that $\lim _{n} f\left(x_{n}\right)=\lim _{n} g\left(x_{n}\right)=t$ for some $t$ in $x$.

(a) If $f$ is continuous at $t, \quad \lim _{n} g f\left(x_{n}\right)=f(t)$.

(b) If $f$ and $g$ are continuous at $t$, then $f(t)=g(t)$ and $f g(t)=g f(t)$.

PROOF. Suppose that $f(t)=g(t)$, and let $x_{n}=t$ for $n$ in N. Then $f\left(x_{n}\right)$, $g\left(x_{n}\right) \rightarrow f(t)$, so that $d(f g(t), g f(t))=d\left(f g\left(x_{n}\right), g f\left(x_{n}\right)\right) \rightarrow 0$ by compatibility. Consequently $d(f g(t), g f(t))=0$ and $f g(t)=g f(t)$, proving 1 .

To prove $2(a)$, note that if $g\left(x_{n}\right) \rightarrow t, f g\left(x_{n}\right) \rightarrow f(t)$ by the continuity of $f$. But if $f\left(x_{n}\right) \rightarrow t$ also, since $d\left(g f\left(x_{n}\right), f(t)\right) \leq d\left(g f\left(x_{n}\right), f g\left(x_{n}\right)\right)+d\left(f g\left(x_{n}\right), f(t)\right)$, the compatibility of $f$ and $g$ require that $d\left(g f\left(x_{n}\right), f(t)\right) \rightarrow 0$; i.e., $g f\left(x_{n}\right) \rightarrow f(t)$.

We prove $2(b)$ by noting that $g f\left(x_{n}\right) \rightarrow f(t)$ by $2(a)$ and the continuity of $f$, whereas $g f\left(x_{n}\right) \rightarrow g(t)$ by the continuity of $g$. Thus $f(t)=g(t)$ by uniqueness of limit, and therefore $g f(t)=f g(t)$ by part 1 . /

\section{COMMON FIXED POINTS FOR $(\varepsilon, \delta)$ CONTRACTIONS.}

Definition 3.1. A pair of self maps $A$ and $B$ of a metric space $(X, d)$ are $(\varepsilon, \delta)$-S,T-contractions relative to maps $S, T: X \rightarrow X$ iff $A(X) C T(X), B(X) C S(X)$, and there is a function $\delta: R^{+} \rightarrow R^{+}$such that $\delta(\varepsilon)>\varepsilon$ for all $\varepsilon$ and for $x, y \varepsilon X$ : 
(i) $\varepsilon \leq d(S x, T y)<\delta(\varepsilon)$ implies $d(A x, B y)<\varepsilon$, and

(ii) $A x=B y$ whenever $S x=T y$.

Note that if $A$ and $B$ are $(\varepsilon, \delta)-S, T$-contractions, then $d(A x, B y) \leq d(S x, T y)$ for all $x, y$, strict inequality holding when $S x \neq T y$. Also observe that in the above definition the pairs $A, S$ and $B, T$ are evaluated at the same points so that order is significant. Consequently, even though $A$ and $B$ are $(\varepsilon, \delta)-S, T-$ contractions, the pair $B, A$ may not be as Example 3.1 will reveal.

Definition 3.2. Let $A, B, S, T$ be self mappings of a set $X$ such that $A(X) C$ $T(X)$ and $B(X) C S(X)$. For $x_{0} \in X$, any sequence $\left\{y_{n}\right\}$ defined by $y_{2 n-1}=T x_{2 n-1}=$ $A x_{2 n-2}$ and $y_{2 n}=S x_{2 n}=B x_{2 n-1}$ for $n \in N$ will be called an S,T-iteration of $x_{0}$ under $A$ and $B$.

The above definition ensures us that for nonempty sets $X$ S,T-iterations will exist since $A(X) C T(X)$ and $B(X) C S(X)$, although the sequences $\left\{y_{n}\right\}$ certainly need not be unique.

LEMIIA 3.1. Let $S$ and $T$ be self maps of a metric space $(X, d)$ and let the pair $A, B$ be $(\varepsilon, \delta)-S, T$-contractions. If $x_{0} \varepsilon X$ and $\left\{y_{n}\right\}$ is an S,T-iteration of $x_{0}$ under $A$ and $B$, then

(a) for each $\varepsilon>0, \varepsilon \leq d\left(y_{p}, y_{q}\right)<\delta(\varepsilon)$ implies $d\left(y_{p+l}, y_{q+1}\right)<\varepsilon$ when $\mathrm{p}$ and $\mathrm{q}$ are of opposite parity,

(b) $d\left(y_{n}, y_{n+1}\right) \rightarrow 0$, and

(c) $\left\{y_{n}\right\}$ is a Cauchy sequence.

PROOF. To prove (a), let $\varepsilon>0$. Since $A$ and $B$ are $(\varepsilon, \delta)-S$, T-contractions, $\varepsilon \leq \mathrm{d}(S \mathrm{x}, \mathrm{Ty})<\delta(\varepsilon)$ implies $\mathrm{d}(\mathrm{Ax}, \mathrm{By})<\varepsilon$.

Now suppose that $\varepsilon \leq d\left(y_{p}, y_{q}\right)<\delta(\varepsilon)$ and that $p$ and $q$ are of opposite parity, say $p=2 n$ and $q=2 m-1$. Then $d\left(y_{p+1}, y_{q+1}\right)=d\left(y_{2 n+1}, y_{2 m}\right)=d\left(A x_{2 n}, B x_{2 m-1}\right)$ and $d\left(y_{p}, y_{q}\right)=d\left(y_{2 n}, y_{2 m-1}\right)=d\left(S x_{2 n}, T x_{2 m-1}\right)$. By the above assumption we thus have $\varepsilon \leq \mathrm{d}\left(\mathrm{Sx}_{2 \mathrm{n}}, \mathrm{Tx}_{2 \mathrm{~m}-1}\right)<\delta(\varepsilon)$, so that (3.1) yields $\mathrm{d}\left(\mathrm{Ax}_{2 \mathrm{n}}, \mathrm{Bx} 2 \mathrm{~m}-1\right)=$ $d\left(y_{p+1}, y_{q+1}\right)<\varepsilon$, as desired.

To see that (b) is true, remember that $d(A x, B y) \leq d(S x, T y)$ for all $x, y$ by the hypothesis on $A, B, S$ and $T$. So if $m$ is even, say $m=2 n, d\left(y_{m}, y_{m+1}\right)=$ $d\left(y_{2 n}, y_{2 n+1}\right)=d\left(B x_{2 n-1}, A x_{2 n}\right) \leq d\left(T x_{2 n-1}, S x_{2 n}\right)=d\left(y_{2 n-1}, y_{2 n}\right)=d\left(y_{m-1}, y_{m}\right)$. Similarly, $d\left(y_{m}, y_{m+1}\right) \leq d\left(y_{m-1}, y_{m}\right)$ if $m$ is odd. Thus the sequence $\left\{d\left(y_{m}, y_{m+1}\right)\right\}$ is nonincreasing and converges to the greatest lower bound of its range which we denote by $r$. Now $r \geq 0$; in fact, $r=0$. Otherwise, part (a) implies $d\left(y_{m+1}, y_{m+2}\right)<r$ whenever $r \leq d\left(y_{m}, y_{m+1}\right)<\delta(r)$ since $m$ and $m+1$ are certainly of opposite parity. But since $\left\{d\left(y_{m}, y_{m+1}\right)\right\}$ converges to $r$, there is a $k$ such that $d\left(y_{k}, y_{k+1}\right)<\delta(r)$ so that $d\left(y_{k+1}, y_{k+2}\right)<r$ - which contradicts the designation of $r$.

To prove part (c) of the Lemma, let $\alpha=2 \varepsilon$ be given. With $r=\delta(\varepsilon)-\varepsilon$, part (a) of the Lemma asserts that

$d\left(y_{p+1}, y_{q+1}\right)<\varepsilon$ whenever $\varepsilon \leq d\left(y_{p}, y_{q}\right)<\varepsilon+r$ and $p$ and $q$ are of opposite parity.

Assume without loss of generality that $r<\varepsilon$. By part (b) of the Lemma we can choose $n_{0} \in N$ such that 


$$
d\left(y_{m}, y_{m+1}\right)<r / 6 \text { for } m \geq n_{0} \text {. }
$$

We now let $q>p \geq n_{0}$ and show that $d\left(y_{p}, y_{q}\right)<\alpha$, thereby proving that $\left\{y_{n}\right\}$ is indeed Cauchy. So suppose that

$$
\mathrm{d}\left(\mathrm{y}_{\mathrm{p}}, \mathrm{y}_{\mathrm{q}}\right) \geq 2 \varepsilon=\alpha \text {. }
$$

In order to show that (3.4) yields a contradiction, we first want an $m>p$ such that

$$
\varepsilon+r / 3<d\left(y_{p}, y_{m}\right)<\varepsilon+r \text { with } p \text { and } m \text { of opposite parity. }
$$

To this end let $k$ be the smallest integer greater than $p$ such that $d\left(y_{p}, y_{k}\right)>\varepsilon+r / 2$. $k$ exists by (3.4) since $r<\varepsilon$. Moreover,

$$
d\left(y_{p}, y_{k}\right)<\varepsilon+(2 r) / 3
$$

For otherwise, $\quad \varepsilon+(2 r) / 3 \leq d\left(y_{p}, y_{k-1}\right)+d\left(y_{k-1}, y_{k}\right)$

$$
<d\left(y_{p}, y_{k-1}\right)+r / 6
$$

since $k-1 \geq p \geq n_{0}$, and therefore

$$
\varepsilon+r / 2<d\left(y_{p}, y_{k-1}\right) .
$$

Since $k-1 \geq p,(3.7)$ implies $p<k-1$. But then (3.7) contradicts the choice of $k$. We thus have

$$
\varepsilon+r / 2<d\left(y_{p}, y_{k}\right)<\varepsilon+(2 r) / 3
$$

Thus, if $p$ and $k$ are of opposite parity we can let $k=m$ in (3.8) to obtain (3.5). If $p$ and $k$ are of like parity, $p$ and $k+1$ are of opposite parity. Moreover, since $d\left(y_{k}, y_{k+1}\right)<r / 6$ by (3.3), the triangle ineauality and (3.8) imply

$$
\varepsilon+r / 3<d\left(y_{p}, y_{k+1}\right)<\varepsilon+(5 r) / 6
$$

In this instance let $m=k+1$.

In any event, by (3.8) and (3.9) we can choose $m$ such that $p$ and $m$ are of opposite parity and (3.5) holds. But then $p, m \geq n_{0},(3.3)$ and (3.5) imply

$$
\begin{aligned}
\varepsilon+r / 3 & <d\left(y_{p}, y_{m}\right) \leq d\left(y_{p}, y_{p+1}\right)+d\left(y_{p+1}, y_{m+1}\right)+d\left(y_{m+1}, y_{m}\right) \\
& <r / 6+d\left(y_{p+1}, y_{m+1}\right)+r / 6 \\
& <r / 3+\varepsilon \text {, by }(3.2) \text { and }(3.5) .
\end{aligned}
$$

This is the anticipated contradiction./

The following lemma highlights the role of compatibility in producing common fixed points.

LEMMA 3.2 Let $S$ and $T$ be self maps of a metric space $(X, d)$ and let $A$ and $B$ be $(\varepsilon, \delta)-S, T$-contractions such that the pairs A,S and $B, T$ are compatible. If there exists $z \in X$ such that $A z=S z$ and $B z=T z$, then $c=T z$ is the unique common fixed point of $A, B, S$ and $T$.

PROOF. The definition of $(\varepsilon, \delta)-S, T$-contractions implies $d(A x, B y)<d(S x, T y)$ if $S x \neq T y$. Thus if $S z \neq T z$, the hypothesis yields the contradiction $d(A z, B z)<$ $d(S z, T z)=d(A z, B z)$. We conclude that $S z=T z=A z=B z$.

Now let $C=T z$ and suppose that $c \neq T c$. Since $T$ and $B$ are compatible and $T z=B z, T B z=B T z$ by Proposition 2.2.1.. But then $d(c, T c)=d(A z, T B z)=d(A z, B T z)$ 
$<d(S z, T T z)=d(c, T c)$, so that $c$ must equal Tc. Similarly, $c=S c$. Since $A$ and $S$ are compatible and $A z=S z=c$, Proposition 2.2.1 implies

$$
A C=A(S z)=S(A z)=S C \text {. }
$$

Likewise, $B C=T c$. Then (3.10) yields: $A c=S c=T C=B C=c . \quad c$ is unique. For if $e$ were another common fixed point of $A, B, S, T$, we would have the contradiction: $d(c, e)=d(A c, B e)<d(S c, T e)=d(c, e) . /$

We can now state and prove our first main result.

THEOREM 3.1. Let $S$ and $T$ be self maps of a metric space $(X, d)$ and let $A$ and $B$ be $(\varepsilon, \delta)-S, T$ - contractions such that the pairs $A, S$ and $B, T$ are compatible. Let $x_{0} \varepsilon X$ and let $\left\{y_{n}\right\}$ by any S,T-iteration of $x_{0}$ under $A$ and B. If $\left\{y_{n}\right\}$ has a cluster point $z$ in $x$, then $\left\{y_{n}\right\}$ converges to $z$, and $T z$ is the unique common fixed point of $A, B, S$ and $T$ provided these functions are continuous at $z$.

PROOF. By Lemma 3.1, $\left\{y_{n}\right\}$ is Cauchy and therefore converges to the cluster point $z$. Then $A x_{2 n}, S x_{2 n}, B x_{2 n-1}, T x_{2 n-1} \rightarrow z$ (See Definition 3.2). Since $A$ and $S$ are compatible and also continuous at $z, A z=S z$ by Proposition 2.2.2.b.. Similarly, $B z=T z$, and the conclusion follows from Lemma 3.2../

The next result follows immediately upon noting that under the given hypothes is, any S,T-iteration of any point under $A$ and $B$ converges to a cluster point since $(X, d)$ is complete.

COROLLARY 3.1. Let $S$ and $T$ be continuous self maps of a complete metric space $(X, d)$ and let $A$ and $B$ be $(\varepsilon, \delta)$-S,T-contractions such that the pairs $A, S$ and $B, T$ are compatible. If $A$ and $B$ are continuous, then $A, B, S$ and $T$ have a unique common fixed point.

Observe that Theorem 1.2. by Park and Bae is a consequence of Corollary 3.1. with $f=S=T$ and $g=A=B$. This follows upon noting that since $g$ is an $(\varepsilon, \delta)-f$ contraction, $d(g(x), g(y)) \leq d(f(x), f(y))$ for all $x, y$ so that the stated continuity of $f$ ensures the continuity of $g$.

The following example of $(\varepsilon, \delta)$-contractions pertains to most of the preceding and as such should be instructive.

EXAMPLE 3.1. Let $x=[1, \infty)$ and $d(x, y)=|x-y|$. Let $S x=\left(x^{4}+1\right) / 2, A x=x^{2}$ $B x=x$ and $T x=\left(x^{2}+1\right) / 2$ for $x$ in $x$. Then $A, B, S$, and $T$ are continuous self maps of the complete metric space $(x, d)$. Since $A 1=S 1=1$ and $(S-A) x=\left(x^{2}-1\right)^{2} / 2$ is increasing on $X, A$ and $S$ are compatible on $X$ by Corollary 2.1. which is clearlyvalid on any connected subset of $R$. However, $A$ and $S$ are not even weakly commutative; consider $\mathrm{x}=2$ for example. On the other hand, $B$ and $T$ are compatible since $B T=T B$.

To see that the hypothesis of Corollary 3.1. is satisfied, we have yet to show that $A$ and $B$ are $(\varepsilon, \delta)-S, T$-contractions. To this end let $\varepsilon>0$. We want a function $\delta: R^{+} \rightarrow R^{+}$such that $\varepsilon \leq|S x-T y|=\left|\left(x^{4}-y^{2}\right) / 2\right|<\delta(\varepsilon)$ implies $|A x-B y|=\left|x^{2}-y\right|<\varepsilon$. Remembering that $x, y \geq 1$, it is easy to show that $\left|\left(x^{4}-y^{2}\right) / 2\right| \geq \varepsilon$ implies that $x^{2}+y \geq(1+\sqrt{2 \varepsilon+1})$. But then $\left|\left(x^{4}-y^{2}\right) / 2\right|<\delta(\varepsilon)$ implies that $\left|x^{2}-y\right|<2 \delta(\varepsilon)\left(x^{2}+y\right)^{-1}<2 \delta(\varepsilon)(1+\sqrt{2 \varepsilon+1})^{-1}$, so that $\left|x^{2}-y\right|<\varepsilon$ if $\delta(\varepsilon)=\varepsilon(1+\sqrt{2 \varepsilon+1}) / 2$. Now $\delta$ so defined for all $\varepsilon>0$ is a continuous mapping from $\mathrm{R}^{+}$into $\mathrm{R}^{+}$such that $\delta(\varepsilon)>\varepsilon$ so that property (i) of Definition 3.1. is satisfied. To confirm property (ii) note that 


$$
|S x-T y|=\left|\left(x^{4}-y^{2}\right) / 2\right|=|A x-B y|\left|\left(x^{2}+y\right) / 2\right|,
$$

which requires that $A x=B y$ when $S x=T y$ since $x, y \geq 1$.

REMARK 3.1. With $A, B, S, T$ defined as in Example 3.1., the pair $A, B$ are $(\varepsilon, \delta)$ $-S, T$-contractions, whereas the pair $B, A$ are not since $|B x-A y|=\left|x-y^{2}\right|=3$ and $|S x-T y|=\left|\left(x^{4}-y^{2}\right) / 2\right|=3 / 2$ with $x=1$ and $y=2$.

In Example 3.1. the function $\delta$ is continuous. Our next result tells us that if we merely require that $\delta$ be lower semicontinuous, $A$ and $B$ in Corollary 3.1. need not be continuous.

THEOREM 3.2. Let $S$ and $T$ be continuous self maps of a complete metric space $(X, d)$ and let $A$ and $B$ be $(\varepsilon, \delta)-S$, T-contractions such that the pairs $A, S$ and $B, T$ are compatible. If $\delta$ is lower semicontinuous, then $A, B, S$ and $T$ have a unique common fixed point.

PROOF. Let $x_{0} \varepsilon X$ and let $\left\{y_{n}\right\}$ be an S,T-iteration of $x_{0}$ under $A$ and $B$. Since $(x, d)$ is complete and since $\left\{y_{n}\right\}$ is Cauchy by Lemma 3.1., $\left\{y_{n}\right\}$ converges to some element $z$ of $X$. Then $A x_{2 n}, S x_{2 n}, B x_{2 n-1}, T x_{2 n-1} \rightarrow z$ (Definition 3.2.). Since $S$ and $T$ are continuous and the pairs $A, S$ and $B, T$ are compatible, Proposition 2.2. and the indicated continuities yield:

$$
T^{2} x_{2 n-1}, B T x_{2 n-1} \rightarrow T z \text { and } S^{2} x_{2 n}, A S x_{2 n} \rightarrow S z .
$$

We assert that $S z=T z$. For suppose that $d(S z, T z)=\varepsilon$ for some $\varepsilon>0$. Since $\delta: \mathrm{R}^{+} \rightarrow \mathrm{R}^{+}$is lower semicontinuous and $\delta(\varepsilon)>\varepsilon$ by definition, there is a neighborhood $N(\varepsilon)$ of $\varepsilon$ such that $\delta(t)>\varepsilon$ for $t \varepsilon N(\varepsilon)$. We can thus choose $t_{0}$ such that $0<t_{0}<\varepsilon<\delta\left(t_{0}\right)$. By (3.12), $d\left(s^{2} x_{2 n}, T^{2} x_{2 n-1}\right) \rightarrow \varepsilon$, and we can therefore choose $n_{0}$ such that $d\left(s^{2} x_{2 n}, T^{2} x_{2 n-1}\right) \varepsilon\left(t_{0}, \delta\left(t_{0}\right)\right)$ for $n \geq n_{0}$. Then the definition of $\delta$ (Definition 3.1) implies that $d\left(A S x_{2 n}, B T x_{2 n-1}\right)<t_{0}$ for $n \geq n_{0}$. But then (3.12) implies that $d(S z, T z) \leq t_{0}<\varepsilon=d(S z, T z)$, the anticipated contradiction.

Now $S z=T z$ implies that $A z=B z$ by Definition 3.1.. Moreover, $A z=T z$ since $d(A z, T z)=\lim _{n} d\left(A z, B T x_{2 n-1}\right) \leq \lim _{n} d\left(S z, T^{2} x_{2 n-1}\right)=d(S z, T z)=0$. By the above we have $A z=B z=S z=T z$, so that $T z$ is the unique common fixed point of $A, B, S$, and $\mathrm{T}$ by Lemma 3.2.. /

COROLLARY 3.2. Let $S$ and $T$ be self maps of a complete metric space $(X, d)$, and let $A, B: X \rightarrow S(X) \cap T(X)$. Suppose that $S$ and $T$ are continuous and that the pairs $A, S$ and $B, T$ are compatible. If there exists $r \in(0,1)$ such that

$$
d(A x, B y) \leq r d(S x, T y) \text { for } x, y \in X,
$$

Then $A, B, S$, and $T$ have a unique common fixed point.

PROOF. Define $\delta$ by $\delta(\varepsilon)=\varepsilon / r$. Then $\delta$ is a continuous self map of $R^{+}$ such that $\delta(\varepsilon)>\varepsilon$. Also, $d(S x, T y)<\delta(\varepsilon)$ implies $d(A x, B y)<r(\varepsilon / r)=\varepsilon$. Thus $A$ and $B$ are $(\varepsilon, \delta)-S, T$-contractions and the hypothesis of Theorem 3.2. is satisfied. /

Corollary 3.2. clearly generalizes Fisher's Theorem 2. in [15]. Moreover, the corollary below generalizes Theorem 1. of [20] by substituting compatibility for commutativity. 
COROLLARY 3.3. Let $S$ and $T$ be continuous self maps of a complete metric space $(X, d)$. Let $\left\{A_{i}: i \varepsilon I\right\}$ be a family of maps $A_{i}: X \rightarrow S(X) \cap T(X)$ compatible with both $S$ and $T$ and let $I$ be any indexing set. If there exists $r \varepsilon(0,1)$ such that

$$
d\left(A_{i} x, A_{j} y\right) \leq r d(S x, T y) \text { for all } x, y \in X \text { and } i \neq j,
$$

then there is a unique point $c \varepsilon X$ such that $c=S c=T c=A_{i}$ for all $i \varepsilon I$.

PRO0F. Let $i, j \in I(i \neq j)$. By corollary 3.2 there is a unique point $c \in X$ such that $c=A_{j} c=A_{j} c=S c=T c$. Now if $k \varepsilon I(i \neq k)$, there is a unique point $d \varepsilon X$ such that $d=A_{i} d=A_{k} d=S d=T d$. Then (3.14) implies:

$$
d(c, d)=d\left(A_{i} c, A_{k} d\right) \leq r d(S c, T d)=d(c, d) \text {. }
$$

Since $r<1$, c must equal $d$ and the conclusion follows. I

REMARK 3.2. The functions in Example 3.1. show that the concept of $(\varepsilon, \delta)$ -S,T-contractions does indeed generalize the relation (3.13) of Corollary 3.2., since in (3.11) we have $|S 1-T y|=|A 1-B y| \mid(1+y) / 2) \mid$ where $(1+y) / 2$ converges to 1 as $y$ approaches 1 from the right; i.e., there exists no $r \varepsilon(0,1)$ such that $|A x-B y| \leq r|S x-T y|$ for all $x, y \geq 1$.

\section{RETROSPECT.}

The preceding results suggest theobvious general question, "To what extent can other fixed point theorems involving commuting maps be strengthened by substituting "compatibility" hypotheses for "commutativity" ?". We however close with more specific

QUESTION 4.1. To what extent is the hypothesis that $\delta$ be lower semi-continuous necessary in Theorem 3.2.?

\section{REFERENCES}

1. JUNGCK, G. Commuting mappings and fixed points. Amer. Math. Monthly 83 (1976) 261-263.

2 . Periodic and fixed points, and commuting mappings. Proc. Amer. Math. Soc. 76(1979) 333-338.

3. Common fixed point theorems for semigroups of maps on L-spaces. Math. Japonica 26(1981) 625-631

4. PARK, S. and BAE, Jong Sook Extensions of a fixed point theorem of Meir and Keeler. Ark. Mat. 19(1981) 223-228.

5. MEIR, A. and KEELER,. E. A theorem on contraction mappings. J. Math. Anal. App1. 2(1969) 526-529.

6. RHOADES, B.E., SESSA, S., KAHN, M.S. and KAHN, M.D. . Some fixed point theorems for Hardy-Rogers type mappings. This Journal 1 No. 1 (1984) 75-87.

7. BASKARAN, R. and SUBRAHMANYAM, P.V. Common fixed points in metrically convex spaces. Jour. Math. Phys. Sci. 18(1984) S65-S70

8. KASAHARA, S. Iff fixed point criterion in L-spaces. Math. Sem. Notes 4 (1976) 205-210.

9. SINGH, S.L. On common fixed points of commuting mappings. Math. Sem. Notes $5(1977)$ 131-134. 
10.

A note on the convergence of a pair of sequences of mappings. Arch. Math. 1, Scripta Fac. Sci. Nat. Ujep Brunensis 15(1979) 47-52.

11. SINGH, S.L. and KuTrestha, Chitra, Coincidence theorems in metric spaces. Ind. J. Phy. Nat. Sci. 2, Sect. B (1982) 19-22.

12. SINGH, S.L. and PANT, B.D. Fixed point theorems for commuting mappings in probalistic metric spaces. Honan Journal 5 (1983) 139-149.

13. DAS, K.M. and NAIK, K.V. Common fixed point theorems for commuting maps on metric spaces. Proc. Amer. Math. Soc. 77(1979) 369-373.

14. RHOADES, B.E., SINGH, S.L. and KULSHRESTHA, Chitra Coincidence theorems for some multivalued mappings. Int. J. of Math. 9, №. 8(1984)429-434.

15. FISHER, Brian Mappings with a common fixed point. Math Sem. Notes 7 (1979) 81-84.

16. PARK, S. A generalization of a theorem of Janos \& Edelstein. Proc. Amer. Math. Soc. 66 (1977) 344-346.

17. CHANG, Cheng Chun On a fixed point theorem of contractive type. Commentarii Mathematici, Univ. Sacti Pauli 32(1983) 15-19

18. CHANG, Shih-Sen A common fixed point theorem for commuting mappings. Proc. Amer. Math. Soc. 83(1981) 645-652.

19. TIVARI, B.M.L. and PANT, B.D. Fixed points of a pair of mappings in probalistic metric spaces. Jnanabaha 13(1983) 13-25.

20. HADZIC, 01ga Common fixed point theorems for family of mappings in complete metric spaces. Math. Japonica 29(1984) 127-134. 


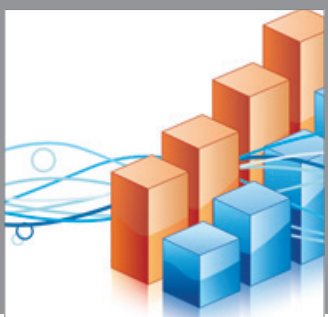

Advances in

Operations Research

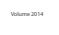

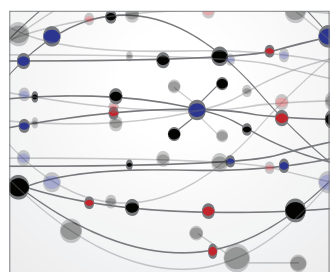

\section{The Scientific} World Journal
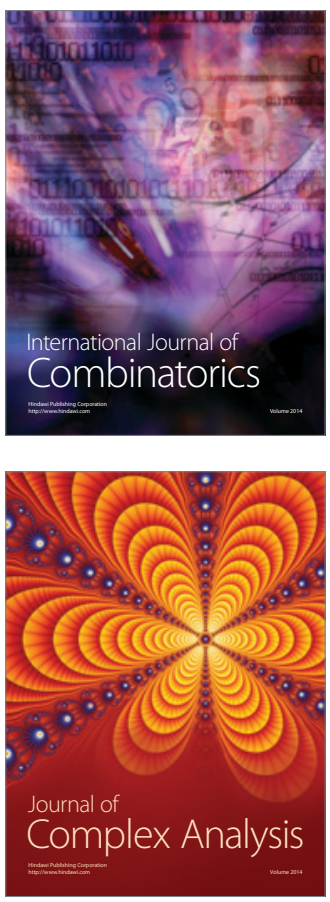

International Journal of

Mathematics and

Mathematical

Sciences
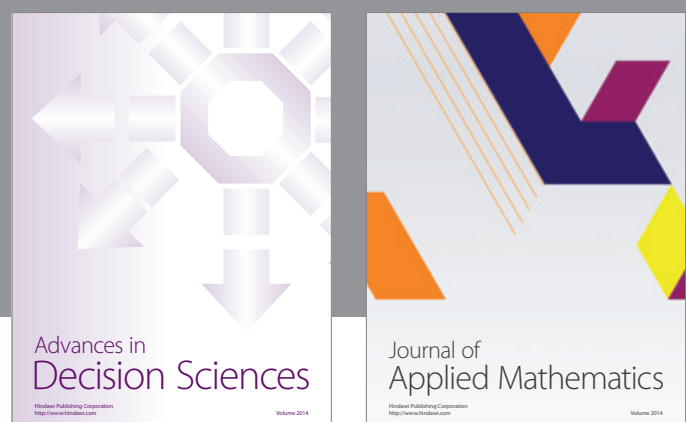

Journal of

Applied Mathematics
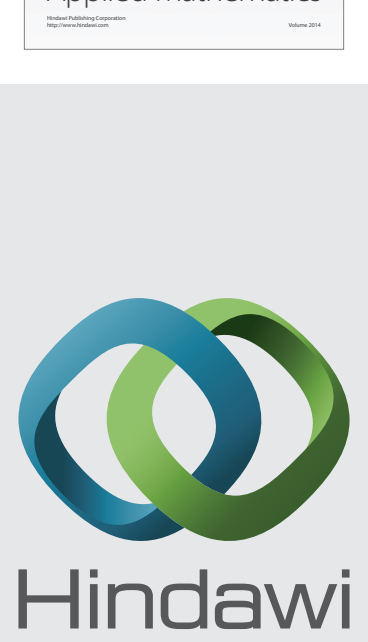

Submit your manuscripts at http://www.hindawi.com
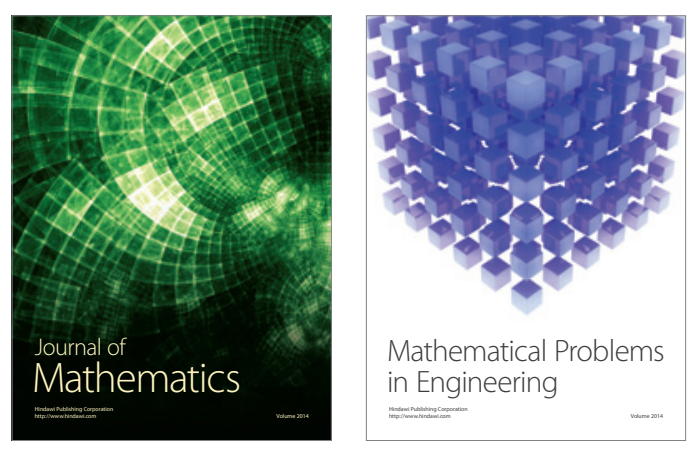

Mathematical Problems in Engineering
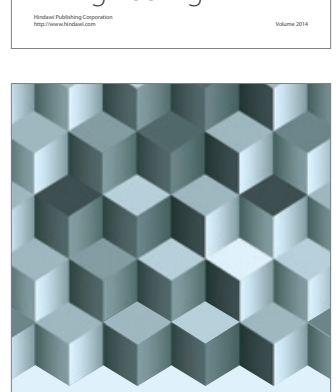

Journal of

Function Spaces
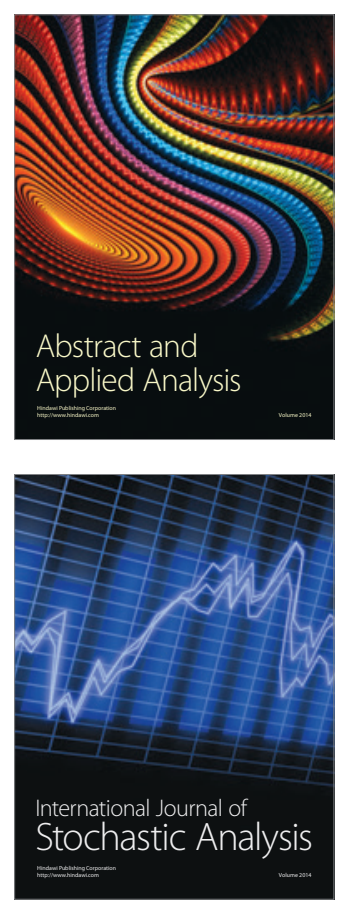

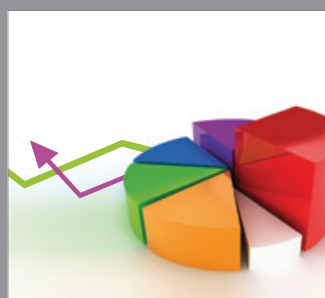

ournal of

Probability and Statistics

Promensencen
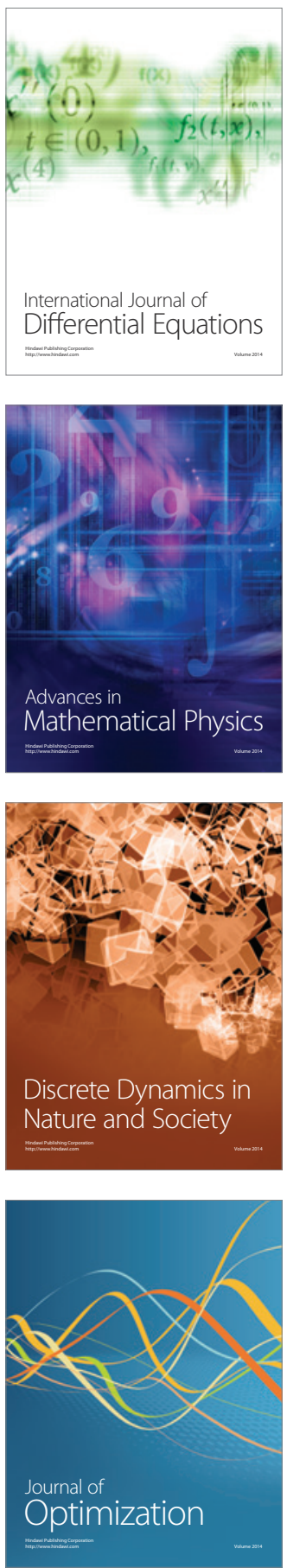